\title{
New Erra with Auto-Haemotherapy
}

\author{
Dina Soliman ${ }^{1,2}$ \\ 1. Rheumatology and Rehabilitation, Ain Shams University, Cairo 11566, Egypt \\ 2. Board Certified in Regenerative Medicine, American Academy of Regenerative Medicine, Denver 80401, USA
}

\begin{abstract}
The active researchers, till now, did not find a curative treatment for many diseases threatening lives of the patients. Proper management and treatment for those patients can give them longer and healthier lives. Over the years, auto-haemotherapy has been used to treat illnesses and diseases such as: arterial circulatory disorders, autoimmune diseases as rheumatoid arthritis, gout, bronchitis, allergies, acne vulgaris, skin eczema, leg ulcers, chronic sinusitis, skin lesions, HIV infection, HCV, Lyme disease, connective tissue diseases, fibromyalgia, asthma, history of heart attack and fungal infections. It helps in cessation of smoking, treatment of drug and alcohol addiction, decreasing the appetite, losing weight in obese patients and speeding up the recovery after a long period of illness. It has also been used in combination with conventional cancer treatment. Auto-haemotherapy is a simple technique where the blood is drawn from a vein and re-injected into a muscle. The blood in the muscle works as a foreign body that will be rejected by the reticulo-endothelial system. It stimulates the immune system and the bone marrow to increase the production of macrophages which are the body's cleaners. The macrophages cleanse everything, they eliminate bacteria, viruses, cancerous cells called neoplastic cells, this is a spring cleaning, and they even eliminate fibrin which is a clotted blood. Auto-haemotherapy is a very powerful technique with a low cost. It requires a syringe and a physician who knows how to withdraw blood from a vein and straight away does an intra-muscular injection with this blood under aseptic condition which increases and stimulates the immune system. Minor auto-haemotherapy technique was applied to 176 patients suffering from different diseases such as: Type I diabetes mellitus, bronchial asthma, chronic sinusitis, allergic conjunctivitis, allergic rhinitis, skin problems (vesicles, eczema, ulcer), finger nail problem (infection, paronychia), acne vulgaris, gastro-intestinal tract problem, lactose intolerance, connective tissue disease, AFS (adrenal fatigue syndrome), myasthenia gravis, fibromyalgia, gout, generalized osteoarthritis, joint osteoarthritis, low back pain with MODIC changes Type 1 on MRI (with or without spinal canal stenosis), auto-immune diseases (ankylosing spondylitis, rheumatoid arthritis), and infectious vaginal discharge. Also it has been applied to patients in order to stop smoking, and to other patients in order to lose weight. Results showed that the patients suffering from the previous different diseases improved significantly after being treated with auto-haemotherapy. In addition, the heavy smokers were able to decrease dramatically the number of cigarettes they were smoking, and the obese patients were able to lose their weight and they had less appetite.
\end{abstract}

Key words: Auto-haemotherapy, Type I diabetes mellitus, treatment of musculoskeletal pain, treatment of auto-immune diseases, blood, intra-muscular injection, immune system stimulation, immune-modulation, virus, bacteria, infection.

\section{Introduction}

Auto-haemotherapy was first described by the French physician Paul Ravaut in 1912 [1]. Autologous blood transfusion is not immunologically neutral and it has an intrinsic immune-modulatory potential even though the basic immune mechanism is still not understood [2].

Auto-haemotherapy, or self-blood therapy, is a technique that involves the withdrawal of blood from the body and the re-injection of the same blood back

Corresponding author: Dina Soliman, M.Sc., research field: non-surgical interventional regenerative orthopedic medicine. into a vein, or through the skin or muscles. Prof. Ravaut [1] used an increasing dose of 1, 2, 3, 4, 5 up to $10 \mathrm{cc}$ of autologous blood to strengthen the immunity, reset the immune system and boost the vitality in order to treat many medical conditions.

The blood in the muscle works as a foreign body that will be rejected by the reticulo-endothelial system. While there is blood in the muscle, the reticulo-endothelial system is being boosted by four times and the maximum boosting finishes only after five days. The normal range of macrophages in the blood is $5 \%$ [1], with auto-haemotherapy this rate raising from $5 \%$ to $22 \%$ over 8 hours and lasting for 5 
days [3]. From the 5th to the 7th day the rate starts to drop again going back to $5 \%$ of the 7 th day. This is why auto-haemotherapy must be repeated every 7 days and can be done for 10,15 or 20 years with no contra-indications [1].

Auto-haemotherapy is a common practice in Europe and South America and is performed to treat pain, ischaemia (a lack of oxygen to the tissues), inflammation and infections. Examples of illnesses and symptoms treated with auto-haemotherapy include arterial circulatory disorders, autoimmune diseases as rheumatoid arthritis, gout, bronchitis, allergies, acne, eczema, leg ulcers, chronic sinusitis, skin lesions, crohn's disease, scleroderma, systemic lupus, ovarian cyst, myomas, thrombocytopenic purpura, multiple sclerosis, cerebral vascular accidents, polymyositis, dermatomyositis, arterial hypertension, recurrent tonsillitis to prevent rheumatic heart diseases, HIV infection, HCV, Lyme disease, connective tissue diseases, fibromyalgia, asthma, history of heart attack and fungal infections. It has also been used in combination with conventional cancer treatment [1], as well as to aid in smoking cessation and to speed up recovery after a long-period illness.

Auto-haemotherapy harmonises the vago-sympathetic system [1] and as a result, it helps people feel more tranquil and helps in reducing the blood pressure.

In sports, Beckenbauer (a German former professional footballer and manager), attributed his physical performance to auto-haemotherapy. He does auto-haemotherapy with 10 cc of blood before each match [1].

Geriatric is the area where auto-haemotherapy should be widely used because this is the time when the immune system is in decline [1]. It could be used in breast feeding women as it will increase the amount of immunoglobulins passing to their babies. It can also be applied to kids if they are compliant and accept being injected [1].

In 1940, a Brazilian professor, Jesse Teixeira, won the biggest prize for surgery with his work published aiming to avoid post-operative infections. He carried out 150 operations of different types, compared to 150 identical operations, with $0 \%$ of post-operative infections in the patients for whom he did auto-haemotherapy prior to the surgery. He injected his patients with $10 \mathrm{cc}$ of their own blood and 5 days later, he injected another 10 cc. To prove his idea, for the same types of operations, he did not do auto-haemotherapy for the patients and there were $20 \%$ cases of infections [1].

Depending on the quantity of blood involved, the technique is described as either minor or major auto-haemotherapy. Minor auto-haemotherapy involves the removal of a small amount of blood, such as 3-10 milliliters, from an individual's vein using a hypodermic needle, which is a hollow needle usually used with a syringe. The blood is then re-injected back into the individual's muscle or through the skin. A typical course of therapy may involve 8-12 injections over a period of 4-6 weeks. In major auto-haemotherapy, a larger quantity of blood, such as 50-250 milliliters, is removed and re-introduced via a vein over a time period of up to 30 minutes, using a standard drip infusion rate or a steady rate. This treatment may be repeated several times per week for a number of weeks [4].

In 1986, Klemparskaya et al. [5] proved in their study that auto-haemotherapy has a stimulating effect on both irradiated and non-irradiated animals, and this was manifested by an increase in resistance to infection, an enhanced production of antibodies to microbes and activated function of cell-mediated immune defense mechanisms.

\section{Patients and Methods}

In this work, 176 patients-while being treated for their musculoskeletal pain, were treated with $10 \mathrm{cc}$ of auto-haemotherapy (5 cc of blood in each buttock), once per week. For patients with Type I diabetes, the treatment was applied for 7 months, and for the other 
patients it was applied for variable durations from 1 month or even less and up to 3 months. Some of the patients wanted to continue after these 3 months because they felt better with auto-haemotherapy and wished to continue. Most of the patients got tired or had a slight increase in their symptoms for 2 or 3 days after the injection, but not all of them. There were 2 patients who had emotional problems with injections, but they were proceeding with the treatment because they were improving. The patients were divided into the following groups:

Group (1): Two brothers with Type I diabetes mellitus: One is 21 years old and his elder brother is 24 years old. They were suffering from diabetes for 12 and 15 years, respectively.

Group (2): Eight patients with bronchial asthma.

Group (3): Ten patients with allergic conjunctivitis.

Group (4): Ten patients with skin eczema.

Group (5): Ten patients with skin pustular-vesicular eruptions.

Group (6): Six patients with finger nail problem (fungal infection).

Group (7): Five patients with lactose intolerance and diarrhea.

Group (8): Twelve patients were treated in order to stop smoking.

Group (9): Nine patients with gastro-intestinal tract problem having hyperacidity and sense of bloating which was considered irritable bowel syndrome.

Group (10): Four patients with chronic fatigue syndrome "adrenal fatigue syndrome".

Group (11): Seven patients with chronic sinusitis.

Group (12): Six patients with allergic rhinitis.

Group (13): Seven obese patients were treated with auto-haemotherapy in order to lose weight.

Group (14): Auto-immune diseases (three patients with ankylosing spondylitis).

Group (15): Three patients with myasthenia gravis.

Group (16): Four patients with fibromyalgia.

Group (17): Eight patients having abnormal vaginal discharge.
Group (18): Four patients with acne vulgaris.

Group (19): Auto-immune diseases (five patients with rheumatoid arthritis).

Group (20): Five patients with connective tissue disease.

Group (21): Eight patients with low back pain having MODIC changes Type I (with or without spinal canal stenosis).

Group (22): Fifteen patients with knee osteoarthritis having on MRI subchondral cysts, bone marrow oedema, areas with high T2 signal intensity in the articular cartilage, meniscus or the cruciate ligaments.

Group (23): Ten patients with generalized osteoarthritis.

Group (24): Fifteen patients with gout.

\section{Results}

The following results have been reported from all the previous groups:

Group (1): The HbA1c of these two brothers was measured prior to the start of the treatment and it was 8.5 and 8.9 respectively (normal range 4.6-6). During the treatment they did not change neither their eating habits nor the units of insulin they were using. After 7 months of continuous weekly treatment, they stopped it as they got bored. HbA1c was re-measured 4 months after the stoppage of the treatment and it was 6.3 and 6.9 respectively. Then another 5 months later, HbA1c was re-measured again and it was 8.5 and 9.5 respectively.

Group (2): Could breath better, they had less wheeze and cough.

Group (3): Had much less redness in the eyes especially on exposure to hot weather. Also they had less lacrimation and itching sensation.

Group (4): Had less itching sensation, and the eczema was much better.

Group (5): The skin was much better which was reflected on their psychological state.

Group (6): The nails improved.

Group (7): Had less diarrhea, less sense of bloating 
and abdominal pain even with ingestion of milk and dairy products.

Group (8): Patients said cigarettes had a bad taste and could decrease the number of cigarettes they were consuming per day.

Group (9): Had less burning sensation in the stomach, less sense of bloating and fullness.

Group (10): Had less pain and more energy. They could perform more activities, they could lose weight better and they felt themselves stronger psychologically and physically.

Group (11): Could breath better, they had less headache and posterior nasal discharge.

Group (12): Had less runny nose, less sneezing and less itching sensation in the nose.

Group (13): Could eat less and they were able to lose weight.

Group (14): Had less pain especially in the hips and back, and their movement improved.

Group (15): Could decrease the dose of mestinon (pyridostigmine bromide), and they could do more activities with more energy without being lethargic.

Group (16): Could sleep better, they had less pain and they were happier.

Group (17): Female patients felt themselves cleaner as there was no discharge.

Group (18): Were so happy as their faces looked relaxed and without acne.

Group (19): Could stop the medications which they were taking for years. They were better on auto-haemotherapy compared to how they were on these medications which have many side effects.

Group (20): Had feelings of well-being. Their generalized body, muscle and joint pain was decreased.

Groups (21-24): Reported a decrease in pain and an improvement in the function of the different body joints, the lower back and lower limbs.

\section{Recommendations}

Auto-haemotherapy is a very easy and simple technique to treat many medical conditions.

Auto-haemotherapy is a very powerful tool to stimulate and re-adjust the immune system of the body, therefore could be used in the treatment of many endocrinological diseases and hormonal unbalance.

Auto-haemotherapy is a very promising technique for treatment of auto-immune diseases instead of the biological treatment and the disease modifying anti-rheumatic drugs that have a lot of side effects on the different body organs.

In cancers, auto-haemotherapy could be used to stimulate the immune system of the body in order to cleanse up the neoplastic cells.

Auto-haemotherapy treats different types of allergies and infections.

Auto-haemotherapy is very useful in the treatment of different gastro-intestinal problems such as helicobacter pylori, lactose intolerance, and chronic diarrhea due to different eitiologies.

Obesity is due to overwhelming connection of the large intestine with pathogenic bacteria which make the patient hungry and eat all the time. Auto-haemotherapy stimulates the immune system of the body to fight and kill these bacteria; therefore it decreases their appetite, changes their eating behavior and helps them in losing weight.

Auto-haemotherpay is the most useful tool in the world to help the smokers in decreasing the number of cigarettes and stopping smoking.

Auto-haemotherapy is the safest and easiest method to treat osteoarthritis.

Auto-haemotherapy is the mystery that medicine missed in the past. It is the power that stimulates the immune system of the body to re-adjust all the body function including the endocrinologial system, the vascular system, the nervous system and the musculoskeletal system.

More research work is needed in the field of auto-haemotherapy to clarify its powerful effectiveness and privileges. 


\section{References}

[1] Auto-haemotherapy - GEOCITIES.ws. www.geocities.ws/autohemoterapiabr/Autohemotherapy_ English_translation_version

[2] Heiss, M., Fraunberger, P., Delanoff, C., Stets, R., and Allgayer, H., et al. 1997. "Modulation of Immune Response by Blood Transfusion: Evidence for a Differential Effect of Allogenic and Autologus Blood in Colorectal Cancer Surgery.” Shock 8 (6): 402-8.
[3] Soliman, D. 2016. Pain Management by Prolotherapy and Perineural Injection Therapy. Germany: Lambert Academic Publishing, 27-34.

[4] De Monte, A., Van Der Zee, H., and Bocci, V. 2005. "Major Ozonated Auto-Haemotherapy in Chronic Limb Ischaemia with Ulcerations.” J. Altern. Complement Med. 11 (2): 363-7.

[5] Klemparskaya, N. N., Shalnova, G. A., Ulanova, A. M., Kuzmina, T. D., and Chuhrov, A. D. 1986. "Immunomodulating Effect of Autohaemotherapy." $J$. Hyg. Epidemiol. Microbil. Immunol. 30 (3): 331-6. 